\title{
Specific Correction of Impaired Acid Hydrolase Secretion in Storage Pool-deficient Platelets by Adenosine Diphosphate
}

\author{
Bruce Lages, Carol A. Dangelmaier, * Holm Holmsen, and Harvey J. Weiss \\ Department of Medicine, Columbia University College of Physicians and Surgeons and St. Luke's-Roosevelt Hospital Center, \\ New York 10019; and*Thrombosis Research Center, Temple University, Philadelphia, Pennsylvania 19140
}

\begin{abstract}
Storage pool-deficient (SPD) platelets, which have decreased amounts of dense-granule and/or $\alpha$-granule constituents, contain normal amounts of lysosomal acid hydrolases, but in some cases exhibit impaired secretion of these enzymes. We examined this impaired secretion response in SPD patients with varying extents of granule deficiencies, and determined the effects of added dense-granule constituents. Acid hydrolase secretion was impaired in patients with severe dense-granule deficiencies, but not in patients with lesser dense-granule deficiencies, including those with $\alpha$-granule deficiencies as well. When dense-granule constituents (ADP, ATP, serotonin, $\mathrm{Ca}^{+2}$, pyrophosphate) were added to gel-filtered platelets, ADP, but none of the other constituents, completely corrected the impairment of thrombin and A23187-induced secretion in SPD platelets. The concentration of ADP required to normalize thrombin-induced secretion varied markedly, from 0.01 to 10 $\mu \mathrm{M}$, among the individual patients. Fixation of platelets with formaldehyde before centrifugation did not prevent the enhancement of secretion by ADP. Excess ATP, which acts as a specific antagonist of ADP-mediated responses, completely blocked this enhancement of secretion in SPD platelets by ADP, and partially inhibited acid hydrolase secretion induced by low, but not high, concentrations of thrombin in normal platelets as well. Treatment of normal platelets with acetylsalicylic acid in vivo, but not in vitro, produced an impairment of acid hydrolase secretion similar in extent to that in SPD platelets, but which could not be completely corrected by added ADP. One possible explanation of these results is that the impairment of acid hydrolase secretion may be secondary to the dense-granule deficiency in SPD platelets, and that secreted ADP may potentiate the lysosomal secretion response in normal platelets as well.
\end{abstract}

\section{Introduction}

The secretion response in human blood platelets encompasses the extrusion of the contents of at least three different types of

A preliminary report of this work was presented at the 7th National Conference on Thrombosis and Haemostasis of the American Heart Association, November 1986 and was published in abstract form (1986. Circulation. 74 [Suppl. II]:423).

Dr. Holmsen's present address is Department of Biochemistry, University of Bergen, Bergen N-5000, Norway. Address reprint requests to Dr. Lages, Department of Medicine, St. Luke's-Roosevelt Hospital Center, 428 West 59th Street, New York, NY 10019.

Received for publication 20 February 1987 and in revised form 17 December 1987.

J. Clin. Invest.

(c) The American Society for Clinical Investigation, Inc.

$0021-9738 / 88 / 06 / 1865 / 08 \quad \$ 2.00$

Volume 81, June 1988, 1865-1872 secretory granules. Small molecules and ions (ATP, ADP, serotonin $[5 \mathrm{HT}],{ }^{1} \mathrm{Ca}^{+2}$, and pyrophosphate $\left[\mathrm{PP}_{\mathrm{i}}\right]$ are secreted from the dense granules, proteins such as fibrinogen, von Willebrand factor, factor $\mathrm{V}, \boldsymbol{\beta}$-thromboglobulin, platelet factor 4 , and platelet-derived growth factor are secreted from $\alpha$-granules, and a variety of acid hydrolytic enzymes are secreted from the lysosomal granules (1). Both quantitative and qualitative differences are found in the patterns of secretion from these granules, primarily in those of lysosomal vs. dense-granule and $\alpha$-granule secretion. Thus, only $30-60 \%$ of the lysosomal enzymes are secreted in response to maximal stimulation, compared with $80-100 \%$ of the dense-granule and $\alpha$ granule contents (1). In addition, only dense-granule and $\alpha$-granule secretion, and not lysosomal secretion, are induced by weak agonists such as ADP or epinephrine (2), and thrombin-induced acid hydrolase secretion, in marked contrast to dense-granule secretion, requires continuous occupancy of the thrombin receptor $(3)$. The dose dependency $(4,5)$, kinetics (6), and energy requirements $(6,7)$ also differ among these three secretory processes when induced by thrombin. These differences in secretion patterns suggest that differences exist in the mechanisms of secretion for each granule, in particular for lysosomal vs. dense-granule and $\alpha$-granule secretion.

In platelets from patients with storage pool deficiency (SPD), the contents of one or more of these secretory granules are reduced or absent (8). We have characterized the types of deficiencies found in SPD (8), which include deficiencies of dense granules ( $\delta$-SPD), as seen in patients with the Hermansky-Pudlak syndrome (9), the Chediak-Higashi syndrome (10-12), and patients without oculocutaneous albinism (8, $13)$; deficiencies of both dense granules and $\alpha$-granules ( $\alpha \delta$ SPD) (8) and selective deficiencies of $\alpha$-granules ( $\alpha$-SPD), as in the gray platelet syndrome (14). The granule deficiencies in SPD do not appear to include the lysosomes, however, since the platelet contents of acid hydrolases were found to be normal in all SPD patients in our studies $(4,8)$ as well as in patients with Chediak-Higashi syndrome (11) and gray platelet syndrome (14).

We found in previous studies (4) that, despite their normal content of these enzymes, platelets from most SPD patients exhibited impaired acid hydrolase secretion in response to low, but not high concentrations of thrombin. A similar abnormality in acid-hydrolase secretion, but not in platelet content, has also been described in the platelets of patients with attentiondeficit disorder (hyperkinesis) (15). The current studies were undertaken to determine the relationship of this secretion defect to the granule deficiencies present in SPD platelets. Our findings that ADP specifically corrected this impairment of acid hydrolase secretion suggest that this defect is secondary to

1. Abbreviations used in this paper: f.c., final concentration; GFP, gel-filtered platelets; $5 \mathrm{HT}$, serotonin; $\mathbf{P P}_{i}$, pyrophosphate; PRP, platelet-rich plasma; SPD, storage pool deficiency. 
the dense-granule deficiency in SPD platelets. We have also obtained evidence that ADP potentiates acid hydrolase secretion in normal platelets as well.

\section{Methods}

Collection of blood, preparation of platelet-rich plasma (PRP) and gelfiltered platelets (GFP). After obtaining informed consent from SPD patients and normal subjects, blood was collected by venipuncture into 0.1 vol of $0.109 \mathrm{M}$ sodium citrate dihydrate. PRP was prepared by centrifugation of citrated whole blood at $1,500 \mathrm{~g}$ for $3 \mathrm{~min}$ at room temperature and was gel-filtered on Sepharose $2 B$ as described previously (16). The platelets were eluted into $\mathrm{Ca}$ - and $\mathrm{Mg}$-free Tyrode's buffer, pH 7.4, containing $1 \mathrm{~g} /$ liter dextrose and $2 \mathrm{~g} /$ /iter lyophilized human serum albumin (Sigma Chemical Co., St. Louis, MO). In some studies, $\mathrm{Ca}$ - and $\mathrm{Mg}$-free Tyrode's without phosphate and containing $0.2 \mathrm{mM} \mathrm{Sr}^{+2}$ was used as the elution fluid. The extents of acid hydrolase secretion were not affected by these alterations.

Thrombin and A23187-induced acid hydrolase secretion. Aliquots of GFP were preincubated with $1.4 \mathrm{mM}$ EDTA at $37^{\circ} \mathrm{C}$ for $3 \mathrm{~min}$ prior to addition of thrombin. In some studies, dense-granule constituents (ATP, ADP, 5HT, $\mathrm{Ca}^{+2}, \mathrm{PP}_{\mathrm{i}}$ or epinephrine) were added to GFP just before or simultaneously with addition of thrombin. Incubation with thrombin $(0.04,0.30$, or $5.0 \mathrm{U} / \mathrm{ml}$ final concentration [f.c.]) was for an additional 3 or $10 \mathrm{~min}$ at $37^{\circ} \mathrm{C}$ without stirring, after which the samples were transferred to an ice bath. The samples were then centrifuged at $17,500 \mathrm{~g}$ for $10 \mathrm{~min}$ at $4^{\circ} \mathrm{C}$. Aliquots of the supernates were extracted by addition of $1.8 \%$ Triton X-100 (1:8 vol/vol) and frozen at $-45^{\circ} \mathrm{C}$ until assayed. Control samples were treated with $0.15 \mathrm{M} \mathrm{NaCl}$ instead of thrombin, and the acid hydrolase activities in these supernates were subtracted from the activities of the thrombin-treated samples. Total platelet acid hydrolase activities were measured in an aliquot of GFP incubated as above with $0.15 \mathrm{M}$ saline and then extracted directly without centrifugation.

For initial studies of the acid hydrolase activities secreted by A23187, aliquots of GFP in Ca- and Mg-free Tyrode's without phosphate and with $0.2 \mathrm{mM} \mathrm{Sr}^{2+}$ were treated with DMSO, 4 or $12 \mu \mathrm{M}$ (f.c.) A23187, added in $0.001 \mathrm{vol}$. Samples were incubated for $3 \mathrm{~min}$ at $37^{\circ} \mathrm{C}$ without stirring, then transferred into tubes containing 0.05 vol $0.1 \mathrm{M}$ EDTA, pH 7.4, in an ice bath, and centrifuged and extracted as described above. Subsequent studies were carried out in the presence of EDTA, as described above for the studies with thrombin.

In studies of the effects of formaldehyde fixation, the aliquots of GFP were split after incubation with thrombin or A23187. One-half of the sample was removed and added to 0.05 vol $0.135 \mathrm{M}$ formaldehyde on ice, and the second half of the sample placed directly in the ice bath. To control for any effects of formaldehyde on the extraction or assay procedures, an equivalent amount of formaldehyde was added to onehalf of the total (uncentrifuged) sample prior to extraction with Triton. Separate studies showed that the presence of formaldehyde had no effect on the acid hydrolase assay itself.

For studies of the recovery of extracellular $\left[{ }^{14} \mathrm{C}\right] \mathrm{ATP}$ after stimulation of GFP, $0.1 \mu \mathrm{M}\left[{ }^{14} \mathrm{C}\right]$ ATP $\left(\left[{ }^{14} \mathrm{C}(\mathrm{U})\right] \mathrm{ATP}, \sim 500 \mathrm{mCi} / \mathrm{mmol}\right.$, NEN Research Products, Boston, MA) was added to each aliquot during the initial 3-min preincubation. After centrifugation, an aliquot of the supernate was extracted with an equal volume of ice-cold ethanol/0.1 M EDTA (9:1) and the adenine nucleotides separated by high-voltage paper strip electrophoresis and counted by liquid scintillation as described previously (16).

For studies of the effects of in vitro aspirin or salicylate incubation on thrombin-induced acid hydrolase secretion in normal subjects, platelets were incubated with $200 \mu \mathrm{M}$ acetylsalicylic acid (ASA) in 0.12 $\mathrm{M} \mathrm{NaHCO}_{3}$, salicylic acid in $\mathrm{NaHCO}_{3}$, or $0.12 \mathrm{M} \mathrm{NaHCO}_{3}$ alone, for 10 min at $37^{\circ} \mathrm{C}$, either in PRP prior to gel filtration or directly in GFP, and then treated with thrombin in the absence or presence of ADP.

Assay of acid hydrolase activities. The activities of $\beta$-hexosaminidase, $\beta$-galactosidase, and $\beta$-glucuronidase were determined by spec- trophotometric assay of $p$-nitrophenol hydrolyzed from the substrates $p$-nitrophenyl- $N$-acetyl- $\beta$-D-glucosaminide, $p$-nitro-phenyl- $\beta$-D-galactopyranoside, and $p$-nitrophenyl- $\beta$-D-glucuronide, respectively, according to previously described methods $(4,17)$. For these studies, however, assay incubation times were increased to $1 \mathrm{~h}$ for $\beta$-hexosaminidase, $3 \mathrm{~h}$ for $\beta$-galactosidase, and $5 \mathrm{~h}$ for $\beta$-glucuronidase. In some studies, acid hydrolase activity was assayed by spectrofluorometry using the appropriate 4-methylumbelliferyl glycoside substrates (17) instead of the $p$-nitrophenol substrates. Activities are expressed as $\mu$ moles substrate hydrolyzed per minute $(1 \mathrm{U})$ per $10^{11}$ platelets.

Radioimmunoassay of thromboxane $B_{2}\left(T \times B_{2}\right)$. Aliquots $(100 \mu \mathrm{l})$ of stimulated platelet suspension were removed after $3 \mathrm{~min}$ and added to $500 \mu$ l of $0.05 \mathrm{M}$ phosphate buffer, $\mathrm{pH} 7.4$, containing $12 \mu \mathrm{M}$ indomethacin and $5 \mathrm{mM}$ EDTA, on ice. After centrifugation at 12,000 $g$ for $2 \mathrm{~min}$, the supernates were frozen at $-45^{\circ} \mathrm{C}$ before radioimmunoassay. $\mathrm{TxB}_{2}$ standards were obtained from the Upjohn Co., Kalamazoo, MI, $\left[{ }^{3} \mathrm{H}\right] \mathrm{TxB}_{2}$ tracer from NEN Dupont, Boston, MA, and $\mathrm{TxB}_{2}$ antibody from Dr. J. B. Smith, Temple University.

Chemicals. Bovine thrombin (Parke-Davis, Detroit, MI) was used without further purification. It was dissolved in $0.15 \mathrm{M} \mathrm{NaCl}$ as a 100 $\mathrm{U} / \mathrm{ml}$ stock, stored at $-45^{\circ} \mathrm{C}$, and diluted to the required concentrations just prior to use. The divalent cation ionophore A23187, a generous gift of Dr. R. L. Hamill, Eli Lilly \& Co., Indianapolis, IN, was dissolved to $20 \mathrm{mM}$ in DMSO, stored at $-45^{\circ} \mathrm{C}$ and diluted just prior to use. All other biochemicals were obtained from Sigma Chemical Co.

Patients. A total of 18 patients with various types of SPD were studied. Nine patients had $\delta$-SPD associated with oculocutaneous albinism (Hermansky-Pudlak syndrome), while six patients had SPD not associated with albinism. Three patients (D.C., S.C., R.C.: mother, son, and daughter) (18) have been characterized as having partial deficiencies of $\alpha$-granules and $\alpha$-granule constituents in addition to densegranule deficiencies (8). Most of these patients have been described in previous studies $(8,13,18,19)$. All of the patients were not included in each of the various studies. Control subjects were hospital and laboratory personnel who denied ingestion of any medication for $7 \mathrm{~d}$ prior to study. In some studies, control subjects were studied before and $2 \mathrm{~h}$ after ingestion of $650 \mathrm{mg}$ of ASA.

Statistical analyses. Statistical significance was determined by the Student's $t$ test, or paired $t$ test where applicable, with appropriate corrections for small sample size and for the possibility of nonequivalent variances in patient and control groups (20).

\section{Results}

Thrombin and A23187-induced acid hydrolase secretion. In accord with previous studies (4), the secretion of $\beta$-hexosaminidase, $\beta$-galactosidase, and $\beta$-glucuronidase was decreased in response to low $(0.04$ and $0.3 \mathrm{U} / \mathrm{ml})$ concentrations of thrombin in SPD platelets (Fig. $1 A$ ). Secretion of $\beta$-hexosaminidase and $\beta$-glucuronidase became normal in the SPD platelets at $5 \mathrm{U} / \mathrm{ml}$ thrombin, as also found previously (4), whereas $\beta$-galactosidase secretion remained slightly ( $P$ $<0.025$, one-tailed $t$ test) decreased (Fig. $1 A$ ). Secretion of these three acid hydrolases was also decreased in SPD platelets, in response to both 4 and $12 \mu \mathrm{M}$ A23187 (Fig. $1 \mathrm{~B}$ ). Higher concentrations of $A 23187$ could not be studied because of the lytic effects of this agonist (19).

Increasing the incubation time from 3 to $10 \mathrm{~min}$ did not increase the extents of secretion obtained in platelets from five SPD patients in response to $0.04 \mathrm{U} / \mathrm{ml}$ thrombin and $12 \mu \mathrm{M}$ A23187 (data not shown). Thus, the impaired secretion is not the result of delayed response.

These patterns of impaired acid hydrolase secretion were observed in platelets from most, but not all, SPD patients. Impaired secretion occurred in patients previously character- 

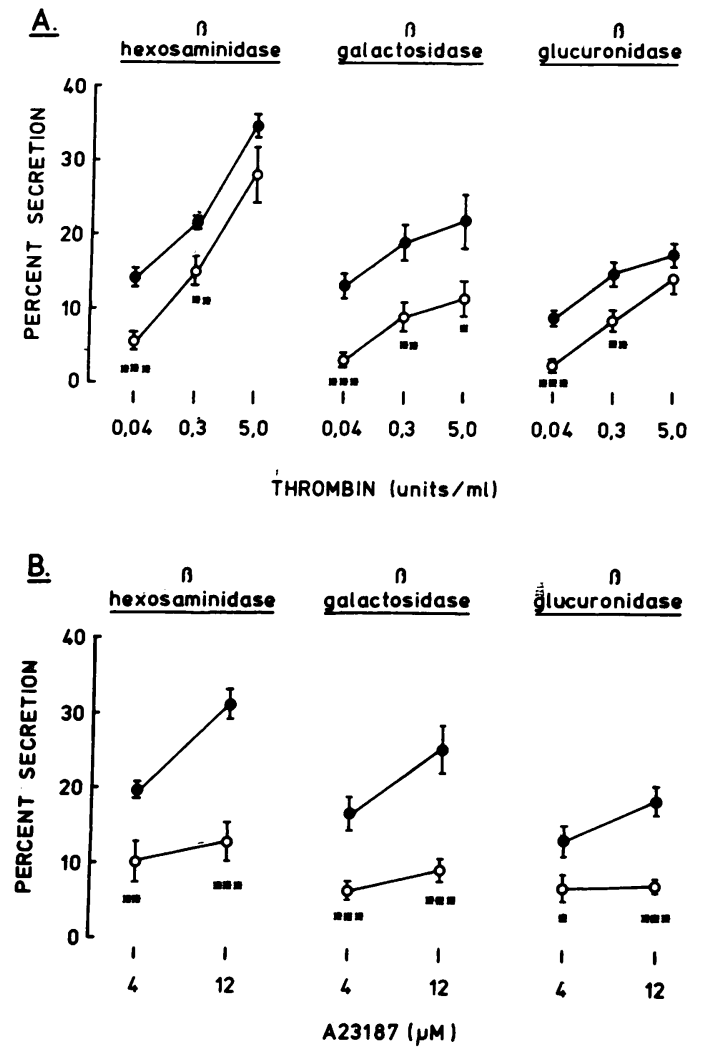

Figure 1. Thrombin and A23187-induced acid hydrolase secretion in SPD vs. normal platelets. GFP at $37^{\circ} \mathrm{C}$ was treated with thrombin or A23187 for $3 \mathrm{~min}$ in the absence of stirring. $(A)$ Secretion induced by $0.04,0.3$, and $5.0 \mathrm{U} / \mathrm{ml}$ thrombin in 15 normal subjects (•) and 10 SPD patients (O). (B) Secretion induced by 4 and $12 \mu \mathrm{M}$ A23187 in 12 normal subjects $(\bullet)$ and 8 SPD patients $(0)$. Values shown are mean \pm SE extents of secretion, expressed as percentages of the total platelet activity measured in Triton X-100-lysed aliquots of GFP, and corrected for the activity in supernates of unstimulated (saline or DMSO-treated) GFP. Significance levels, for SPD vs. normal, are given as: NS, not significant; ${ }^{*} P<0.025 ;{ }^{* *} P<0.005 ;{ }^{* * *} P<0.001$.

ized as having deficiencies of dense granules only $(\delta$-SPD) (8), and was particularly evident in the $\delta$-SPD albino patients (Table I). These patients exhibit the most severe dense-granule deficiencies, as indicated by a virtual absence of secreted ATP and ADP in response to $5 \mathrm{U} / \mathrm{ml}$ thrombin (19). In contrast, little or no impairment of acid hydrolase secretion was ob-

Table I. Comparison of Acid Hydrolase Secretion and Maximal Secretable ADP in Gel-filtered SPD Platelets

\begin{tabular}{lcc}
\hline \multicolumn{1}{c}{ SPD type } & $\begin{array}{c}\text { Thrombin }(0.04 \mathrm{U} / \mathrm{ml}) \text {-induced } \\
\beta \text {-hexosaminidase secretion }\end{array}$ & $\begin{array}{c}\text { Maximal } \\
\text { secretable ADP }\end{array}$ \\
\hline & $\%$ of normal & $\%$ of normal \\
$\delta$-SPD, albino $(n=6)$ & $42 \pm 10$ & $0.2 \pm 0.4$ \\
$\delta$-SPD, nonalbino $(n=1)$ & 67 & 25 \\
$\alpha \delta$-SPD & $106 \pm 17$ & $14 \pm 6$ \\
\hline
\end{tabular}

Values shown are means \pm SD for $\delta$-SPD, albino and $\alpha \delta$-SPD patients. Maximal secretable ADP was measured as the amount released in response to $5 \mathrm{U} / \mathrm{ml}$ thrombin. ADP was assayed by the firefly luciferase technique as described by Lages et al. (19).

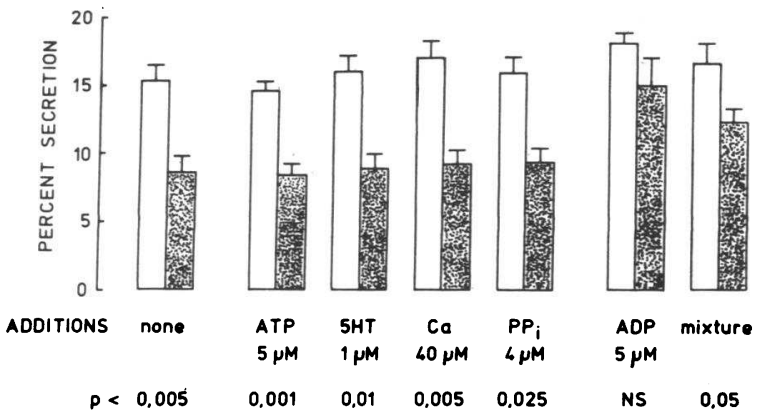

Figure 2. Effect of added dense-granule constituents on thrombin-induced $\beta$-hexosaminidase secretion in SPD and normal GFP. The dense-granule constituents, at the concentrations indicated, were added to GFP at $37^{\circ} \mathrm{C}$ just before addition of $0.04 \mathrm{U} / \mathrm{ml}$ thrombin for $3 \mathrm{~min}$. The bars labeled "mixture" indicate secretion obtained in the presence of all the constituents, at the concentrations indicated for the individual substances. Values shown are mean $\pm S E$ extents of secretion in GFP from four SPD patients (darkened bars) and seven normal subjects (open bars). The $P$ values are given for SPD vs. normal.

served in one $\delta$-SPD patient with a less severe dense-granule deficiency, and in three related patients with combined deficiencies of dense granules and $\alpha$-granules ( $\alpha \delta$-SPD), in whom the dense-granule deficiencies are also less severe than those in the $\delta$-SPD albino patients (Table I). These patterns of normal and abnormal secretion thus suggest that $\alpha$-granule constituents do not play a role in acid hydrolase secretion, but that a substance or substances secreted from dense granules may be involved in this secretion response.

Effect of added (extracellular) dense-granule constituents on thrombin-induced acid hydrolase secretion. To examine this possible involvement of dense-granule substances in acid hydrolase secretion, the secretion of $\beta$-hexosaminidase induced by a low concentration $(0.04 \mathrm{U} / \mathrm{ml})$ of thrombin was measured in normal and SPD platelets in the presence of added densegranule constituents (ATP, ADP, $5 \mathrm{HT}, \mathrm{Ca}^{+2}$, and $\mathrm{PP}_{\mathrm{i}}$ ) at concentrations approximating those obtained extracellularly after maximal secretion in normal platelets. As shown in Fig. 2, none of these dense-granule constituents had any effect on $\beta$-hexosaminidase secretion in normal platelets. However, ADP, but none of the other constituents, completely corrected the impaired secretion response seen in SPD platelets. When ADP was added together with the other dense-granule constituents, secretion was also significantly increased in the SPD platelets compared with the extent in the absence of these constituents $(12.2 \pm 1.05 \mathrm{SE}$ vs. $8.6 \pm 1.2, P<0.025$, paired $t$ test), but remained slightly less than that obtained in normal platelets.

Since these studies were performed in the presence of 1.4 mM EDTA to eliminate any effects of platelet-platelet interactions on the secretion response, the effects of $\mathrm{Ca}^{+2}$ were therefore also examined in the absence of EDTA. The extent of $\beta$-hexosaminidase secretion in SPD platelets was increased when $40 \mu \mathrm{M} \mathrm{Ca}^{+2}$ was added in the absence of EDTA, but was also increased in the absence of EDTA without addition of $\mathrm{Ca}^{+2}$ (Table II). However, similar increases were also seen in normal platelets under the same conditions. Thus, this enhancement of $\beta$-hexosaminidase secretion by $\mathrm{Ca}^{+2}$ appears to represent a more general influence on the secretory response, 
Table II. Effect of $\mathrm{Ca}^{+2}$ on Thrombin $(0.04 \mathrm{U} / \mathrm{ml})$-induced $\beta$-Hexosaminidase Secretion in the Absence of EDTA

\begin{tabular}{ccc}
\hline & \multicolumn{2}{c}{$\begin{array}{c}\text { Relative extent of } \\
\beta \text {-hexosaminidase secretion }\end{array}$} \\
\cline { 2 - 3 } Conditions & $\operatorname{SPD}(n=5)$ & Normal $(n=5)$ \\
\hline$+1.4 \mathrm{mM}$ EDTA & 100 & 100 \\
$-1.4 \mathrm{mM} \mathrm{EDTA}$ & $117 \pm 6$ & $141 \pm 11$ \\
& $(<0.05)^{*}$ & $(<0.01)$ \\
$+40 \mu \mathrm{M} \mathrm{CaCl}_{2}$ & $135 \pm 16$ & $154 \pm 15$ \\
& $(<0.05)$ & $(<0.025)$ \\
\hline
\end{tabular}

GFP with or without $1.4 \mathrm{mM}$ (f.c.) EDTA or with $40 \mu \mathrm{M}$ (f.c.) $\mathrm{CaCl}_{2}$ was incubated for $3 \mathrm{~min}$ at $37^{\circ} \mathrm{C}$ without stirring and then for an additional $3 \mathrm{~min}$ with thrombin $(0.04 \mathrm{u} / \mathrm{ml})$. Incubation was terminated by transfer to an ice bath, the platelets were removed by centrifugation, and the supernates were extracted with $1.8 \%$ Triton $\mathrm{X}-100$ and assayed for $\beta$-hexosaminidase activity. Extents of secretion are expressed as mean $( \pm \mathrm{SE})$ percentages of the values obtained in the presence of $1.4 \mathrm{mM}$ EDTA; which were $10.2 \pm 2.2$ for SPD patients and $21.0 \pm 1.2$ for normals. $P$ values given in parentheses.

* $P$ values relative to secretion in the presence of $1.4 \mathrm{mM}$ EDTA.

rather than a specific correction of the impairment in SPD platelets as seen with ADP in the presence of EDTA.

Epinephrine is also present in platelet dense granules, although at concentrations several thousandfold lower than ATP, ADP, or 5HT (21). Since epinephrine has been shown to enhance aggregation responses in functionally inhibited platelets, including SPD platelets (22), we also tested the effect of epinephrine on acid hydrolase secretion. As shown in Fig. 3, this secretion response was increased in SPD platelets in the presence of 5-10 $\mu \mathrm{M}$ epinephrine, but was unchanged when epinephrine was added at submicromolar or nanomolar levels, corresponding to the amounts present in platelets or normally present in plasma (21).

Effects of ADP on A23187-induced acid hydrolase secretion in SPD platelets. Acid hydrolase secretion induced by $12 \mu \mathrm{M}$ A23187 was also normalized in SPD platelets in the presence of $5 \mu \mathrm{M}$ ADP (Fig. 4). In these and some other studies (see below), ADP and ATP were both present at concentrations corresponding to the maximal amounts secreted from platelet dense granules ( $5 \mu \mathrm{M}$ each). However, as for the thrombininduced response (Fig. 2), subsequent studies showed that

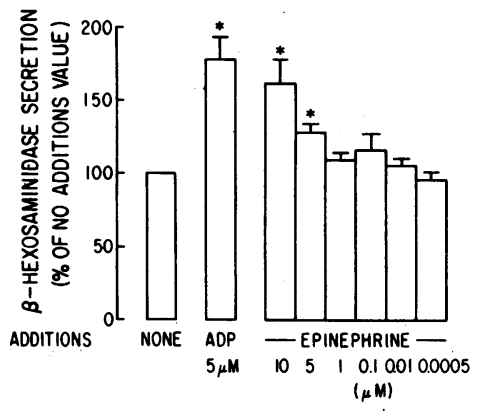

Figure 3. Effect of epinephrine vs. ADP on $\beta$-hexosaminidase secretion induced by $0.04 \mathrm{U} / \mathrm{ml}$ thrombin in SPD platelets. GFP was treated with ADP or epinephrine, at the concentrations indicated and secretion induced by $0.04 \mathrm{U} / \mathrm{ml}$ thrombin as described in the legend to Fig. 2. Secretion is expressed relative to

the value obtained without addition of ADP or epinephrine, which in these studies was $7.5 \pm 0.6 \%$ (SE) $(n=5)$, and is given as mean $\pm \mathrm{SE}$. The asterisks indicate significance levels of $P<0.05$ relative to the no additions value, as assessed by a one-tailed paired $t$ test.

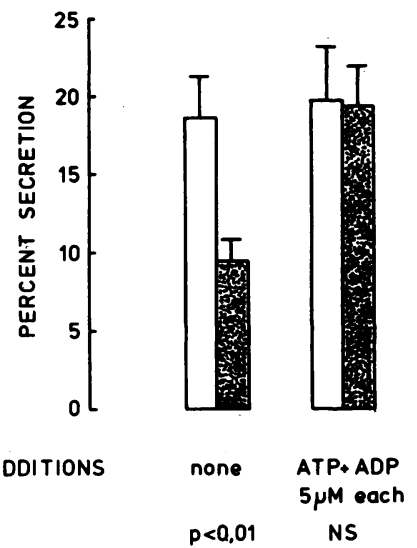

Figure 4. Enhancement of A23187-induced $\beta$-hexosaminidase secretion in SPD GFP in the presence of ADP + ATP Addition of ADP + ATP (5 $\mu \mathrm{M}$ each) to GFP and treatment with A23187 was performed as for the thrombin studies described in Fig. 2. The values are mean $\pm S E$ extents of secretion in GFP from four SPD patients (darkened bars) and nine normal subjects (open bars). The correction of the secretion response in SPD platelets in the presence of

ADP + ATP is due to ADP, since, in separate studies, ATP alone was found to have no effect on the secretion response.

ATP alone, at these concentrations, did not alter the extent of A23187-induced secretion (data not shown). Thus the correction of the impaired secretion response seen in Fig. 4 is also attributed to the effects of added ADP.

Enhancement of secretion by ADP in formaldehyde-fixed $S P D$ platelets. Platelet secretion can also be induced during centrifugation by close-cell contact mechanisms (23),. and these effects can be prevented by treatment with formaldehyde prior to centrifugation (24). To determine whether ADP might enhance acid hydrolase secretion via effects on such mechanisms, secretion was measured in platelets treated with formaldehyde after stimulation with thrombin or A23187 but prior to centrifugation (Table III). Formaldehyde treatment decreased the extents of secretion in both SPD and normal platelets, compared with the values obtained in the absence of formaldehyde (cf. Figs. 2 and 4), indicating a contribution of close-cell contact mechanisms with both agonists. This decrease, and hence the relative contribution of these mechanisms, was greater for A23187 than for thrombin. However, both thrombin and A23187-induced acid hydrolase secretion

Table III. Effect of ADP + ATP on Acid Hydrolase Secretion in SPD and Normal Platelets Fixed with Formaldehyde before Centrifugation

\begin{tabular}{ccrr}
\hline & & \multicolumn{2}{c}{$\begin{array}{c}\text { Percent } \beta \text {-hexosaminidase } \\
\text { secretion }\end{array}$} \\
\cline { 3 - 4 } Agonist & $\begin{array}{c}\text { ADP + ATP } \\
(5 \mu \mathrm{M} \text { each })\end{array}$ & $\operatorname{SPD}(n=4)$ & Normals $(n=6)$ \\
\hline Thrombin $(0.04 \mathrm{U} / \mathrm{ml})$ & - & $5.1 \pm 1.2^{*}$ & $11.0 \pm 1.5$ \\
& + & $10.4 \pm 0.4$ & $12.0 \pm 1.5$ \\
A23187 $(12 \mu \mathrm{M})$ & - & $2.8 \pm 0.6$ & $5.3 \pm 1.4$ \\
& + & $11.5 \pm 3.8$ & $7.0 \pm 2.4$
\end{tabular}

GFP at $37^{\circ} \mathrm{C}$ was treated with thrombin or $\mathrm{A} 23187$ in the absence of stirring for $3 \mathrm{~min}$ in the absence or presence of ADP + ATP, $5 \mu \mathrm{M}$ each, added just before the agonist. $0.05 \mathrm{vol}$ of $2.7 \mathrm{M}$ formaldehyde (i35 $\mathrm{mM}$ f.c.) was then added to each sample before centrifugation, and $\beta$-hexosaminidase activity was measured in Triton X-100 extracts of the supernates. The values shown are mean $\pm S E$ extents of secretion.

${ }^{*} P<0.01$ vs. normal value. 


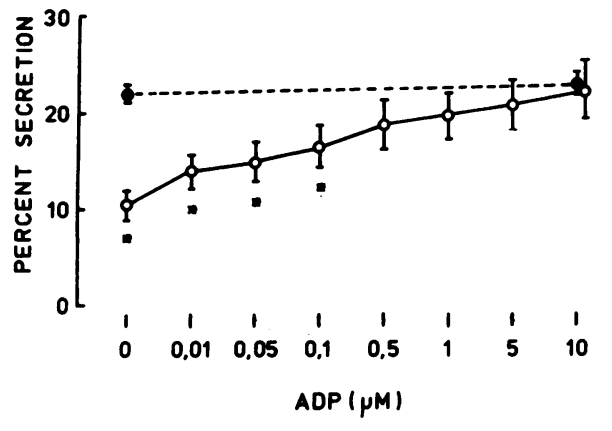

Figure 5. Concentration dependence of ADP enhancement of thrombin-induced acid hydrolase secretion in SPD platelets. Platelets were treated with ADP, at the concentrations indicated, and $0.04 \mathrm{U} / \mathrm{ml}$ thrombin as described for Fig. 2. Values shown are the mean $\pm S E$ extents of $\beta$-hexosaminidase secretion in GFP from five SPD patients $(0)$ and five normal subjects (๑). The asterisk identifies mean SPD values significantly $(P<0.025)$ less than the mean normal values.

were still completely corrected in the presence of ADP plus ATP when formaldehyde was added after stimulation. (As noted above, this correction is attributed specifically to ADP.) Thus, ADP acts directly on the agonist-induced stimulation occurring in suspension rather than on close-cell contact-mediated activation occurring during centrifugation.

Concentration dependence of ADP enhancement of acid hydrolase secretion. The effects of varying concentrations of ADP, from 0.01 to $10 \mu \mathrm{M}$, on the acid hydrolase secretion response were studied in platelets from five SPD patients. As shown in Fig. 5, the mean extent of $\beta$-hexosaminidase secretion induced by $0.04 \mathrm{U} / \mathrm{ml}$ thrombin increased in a concentration-dependent manner over the range of ADP concentrations tested. At $0.5 \mu \mathrm{M} \mathrm{ADP}$ and above, the mean extent of secretion in the patients did not differ significantly from that in five normal subjects. Secretion was significantly increased in the SPD platelets even at $0.01 \mu \mathrm{M}$ ADP, compared to the extent obtained in the absence of added ADP $(P<0.01$, onetailed paired $t$ test).

Among the individual patients, however, the sensitivity to ADP varied substantially. Fig. 6 shows the ADP dose-response data in the individual patients compared to the mean \pm 2 SD ranges of the five normal subjects, In the patient with the

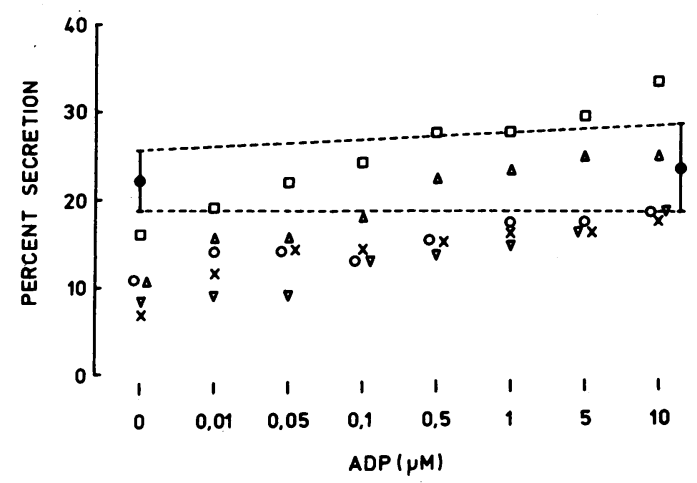

Figure 6. Concentration dependence of ADP enhancement of thrombin-induced acid hydrolase secretion in individual SPD patients. The individual values of extent of $\beta$-hexosaminidase secretion in the same five SPD patients as shown in Fig. 4 are plotted vs. the mean \pm 2 SD range for the five normal subjects shown in Fig. 4. highest level of secretion in the absence of ADP, addition of $0.01 \mu \mathrm{M}$ ADP was sufficient to bring the extent of secretion into the normal range, whereas in three other patients with more severely impaired secretion responses, the extents of secretion did not reach the normal range until $10 \mu \mathrm{M}$ ADP was present. Thus, a considerable degree of heterogeneity in platelet sensitivity to the effects of ADP exists among the SPD patients. In none of the patients or normal subjects was ADP alone, in the absence of thrombin, capable of inducing $\beta$-hexosaminidase secretion.

Recovery of labeled nucleotides from thrombin- and A23187-stimulated SPD platelets. Previous observations have shown that small amounts of ATP and ADP are secreted from the platelets of some SPD patients $(19,25)$. To assess the possibility that degradation of secreted nucleotides, rather than just insufficient amounts of secreted ADP, might also contribute to the lysosomal secretion defects in some patients, $0.1 \mu \mathrm{M}\left[{ }^{14} \mathrm{C}\right]-$ ATP was added to GFP before stimulation and the radioactivity remaining as ATP and ADP after stimulation was quantitated. Comparable extents of recovery of $\left[{ }^{14} \mathrm{C}\right] \mathrm{ATP}$ radioactivity, ranging from $91 \%$ to $108 \%$ of initial radioactivity, were obtained in SPD $(n=3)$ and normal $(n=4)$ platelets after thrombin or A23187 stimulation. The recovery of radioactivity in $\left[{ }^{14} \mathrm{C}\right] \mathrm{ADP}$ was also similar in SPD and normal platelets under these conditions. Thus, no evidence could be found that degradation of nucleotides after their secretion might also contribute to the impaired lysosomal secretion response in SPD platelets.

Inhibition of ADP effects by excess ATP. The dependence of the enhancement of acid hydrolase secretion in SPD platelets on interactions of ADP with its membrane receptor was examined using excess $(500 \mu \mathrm{M})$ ATP as a specific antagonist of ADP-induced responses (26). Such high concentrations of ATP are required to observe its competitive inhibition of ADP-induced responses (26); accordingly, little or no inhibition was seen when ATP was present with ADP in equivalent micromolar levels (cf. Figs. 2 and 4, and Table III). As shown in Table IV, the ability of $10 \mu \mathrm{M}$ ADP to increase the throm-

Table IV. Alteration of ADP Effects on Acid Hydrolase Secretion in SPD and Normal Platelets by Excess $(500 \mu M)$ ATP

\begin{tabular}{llccc}
\hline & & \multicolumn{2}{c}{$\begin{array}{c}\beta \text {-Hexosaminidase } \\
\text { secretion }\end{array}$} \\
\cline { 3 - 4 } Platelet source & \multicolumn{1}{c}{ Additions to GFP } & $\begin{array}{c}0.04 \mathrm{U} / \mathrm{ml} \\
\text { thrombin }\end{array}$ & $\begin{array}{c}1.0 \mathrm{U} / \mathrm{ml} \\
\text { thrombin }\end{array}$ \\
\hline \multirow{4}{*}{$\mathrm{SPD}(n=3)$} & None & $\%$ & $\%$ \\
& $10 \mu \mathrm{M}$ ADP & $7.6 \pm 0.4$ & - \\
& $10 \mu \mathrm{M}$ ADP $+500 \mu \mathrm{M}$ ATP & $5.7 \pm 0.3^{*}$ & - \\
Normals $(n=3)$ & None & $21.0 \pm 3.0$ & $30.2 \pm 1.6$ \\
& $10 \mu \mathrm{M}$ ADP & $18.1 \pm 2.3$ & $30.6 \pm 1.4$ \\
& $10 \mu \mathrm{M}$ ADP $+500 \mu \mathrm{M}$ ATP & $10.0 \pm 1.9^{*}$ & $26.6 \pm 2.2$
\end{tabular}

GFP at $37^{\circ} \mathrm{C}$ was treated with 0.04 or $1.0 \mathrm{U} / \mathrm{ml}$ thrombin in the absence of stirring for $3 \mathrm{~min}$, in the absence of any additions, or in the presence of $10 \mu \mathrm{M}$ ADP, or $10 \mu \mathrm{M}$ ADP plus $500 \mu \mathrm{M}$ ATP, added just before thrombin. $\beta$-Hexosaminidase activity was measured in Triton X-100 extracts of the supernates obtained after centrifugation. Values shown are mean $\pm S E$ extents of secretion.

* $P<0.05$ relative to no additions value. 
Table V. Effect of Epinephrine on Thrombin $(0.04 \mathrm{U} / \mathrm{ml})$-induced $\beta$-hexosaminidase Secretion in Normal Platelets Inhibited with Excess $(500 \mu M)$ ATP

\begin{tabular}{cc}
\hline \multicolumn{1}{c}{ Additions to GFP } & $\beta$-Hexosaminidase secretion \\
\hline & $\%$ of control \\
None (control) & 100 \\
$500 \mu \mathrm{M}$ ATP & $60 \pm 5$ \\
$500 \mu \mathrm{M} \mathrm{ATP}+$ epinephrine & \\
$10 \mu \mathrm{M}$ & $92 \pm 8^{*}$ \\
$5 \mu \mathrm{M}$ & $70 \pm 7^{*}$ \\
$1 \mu \mathrm{M}$ & $63 \pm 8$ \\
$0.1 \mu \mathrm{M}$ & $59 \pm 5$ \\
$0.01 \mu \mathrm{M}$ & $55 \pm 7$ \\
\hline
\end{tabular}

Additions of ATP and epinephrine were made as described in the legend to Table IV. Values are means \pm SE for four normal subjects. $* P<0.05$ relative to $500 \mu \mathrm{M}$ ATP value, using a one-tailed paired $t$ test.

bin-induced acid hydrolase secretion response in SPD platelets was completely abolished by $500 \mu \mathrm{M}$ ATP. Furthermore, in normal platelets, whereas ADP again had no effect on the secretion response, excess ATP partially inhibited secretion induced by $0.04 \mathrm{U} / \mathrm{ml}$ thrombin, but did not affect the response to a higher $(1.0 \mathrm{U} / \mathrm{ml})$ thrombin concentration. This inhibition by excess ATP could not be reversed by $10 \mu \mathrm{M}$ ADP, but was reversed, in part, by addition of 5-10 $\mu \mathrm{M}$ epinephrine (Table V). However, as noted above relative to its effects in SPD platelets, this reversal of inhibition by epinephrine was seen only at micromolar levels, and did not occur at concentrations closer to physiological levels.

Effects of ASA in vivo and in vitro on acid hydrolase secretion. The effects of exposure of normal platelets to ASA, both in vivo and in vitro, on thrombin-induced acid hydrolase secretion were compared with the impairment of this secretion response seen in SPD platelets. Incubation of normal platelets with ASA in vitro, either in PRP or GFP, had no effect on the extent of acid hydrolase secretion (Table VI), in accord with previous observations (4).

In marked contrast, exposure of normal platelets to ASA in vivo caused a significant decrease in the extent of $\beta$-hexosaminidase secretion. A similar pattern of inhibition of acid hydrolase secretion by ASA in vivo, but not in vitro, has also been observed in previous studies (27). Addition of $10 \mu \mathrm{M}$ ADP increased the extent of secretion after in vivo ASA treatment, but, unlike its effect on SPD platelets, failed to normalize the response completely.

The differences seen in the effects of in vivo vs. in vitro ASA treatment on acid hydrolase secretion were not due to differences in inhibition of $\mathrm{TxA}_{2}$ formation. Under all conditions of incubation with ASA, TxA $\mathrm{A}_{2}$ formation, measured by radioimmunoassay of $\mathrm{TxB}_{2}$, was inhibited by $>90 \%$. In addition, incubation of platelets in vitro with $200 \mu \mathrm{M}$ salicylate did not alter the extent of $\beta$-hexosaminidase secretion (data not shown).

\section{Discussion}

These studies demonstrate that the impairment of acid hydrolase secretion in SPD platelets, documented here and in earlier
Table VI. Effect of Incubation with Aspirin In Vitro or In Vivo on Thrombin-induced Acid Hydrolase Secretion in Normal Platelets

\begin{tabular}{|c|c|}
\hline Incubation & $\begin{array}{l}\text { Thrombin }(0.04 \text { units/ml)-induced } \\
\beta \text {-hexosaminidase secretion }(\%)\end{array}$ \\
\hline \multicolumn{2}{|l|}{ In vitro } \\
\hline No ASA & $21.9 \pm 1.6$ \\
\hline $\begin{array}{l}200 \mu \mathrm{M} \text { ASA, } 10 \mathrm{~min}, 37^{\circ} \\
\text { added to PRP }\end{array}$ & $21.3 \pm 3.3$ \\
\hline $\begin{array}{l}200 \mu \mathrm{M} \text { ASA, } 10 \mathrm{~min}, 37^{\circ} \\
\text { added to GFP }\end{array}$ & $19.3 \pm 1.8$ \\
\hline \multicolumn{2}{|l|}{ In vivo } \\
\hline Pre-ASA & $22.1 \pm 1.4$ \\
\hline $2 \mathrm{~h}$ after $650 \mathrm{mg} \mathrm{ASA}$ & $13.1 \pm 3.0^{*}$ \\
\hline After ASA, $+10 \mu \mathrm{M}$ ADP & $16.3 \pm 3.1^{*}$ \\
\hline
\end{tabular}

Platelets were exposed to ASA in vitro by incubation of either PRP before gel filtration, or GFP with $200 \mu \mathrm{M}$ ASA in $0.12 \mathrm{M} \mathrm{NaHCO}_{3}$ for $10 \mathrm{~min}$ at $37^{\circ} \mathrm{C}$, or in vivo by ingestion of $2 \times 325 \mathrm{mg}$ ASA $2 \mathrm{~h}$ prior to blood collection. In vitro no ASA samples were incubated with $0.12 \mathrm{M} \mathrm{NaHCO}_{3}$ alone. In vivo control samples were obtained before aspirin ingestion. GFP was treated with thrombin in the presence or absence of ADP and $\beta$-hexosaminidase secretion measured as described in Tables II and III. Values shown are mean \pm SE extents of secretion measured in five normal subjects for the in vitro studies and in three normal subjects for the in vivo studies.

$* P<0.05$ vs. pre-ASA value.

work (4), can be completely corrected by exogenous ADP. This effect was specific to ADP in that correction was obtained on addition of ADP alone but not on addition of any of the other dense-granule constituents-ATP, $5 \mathrm{HT}, \mathrm{Ca}^{+2}$, or $\mathrm{PP}_{\mathrm{i}}-$ alone. Although epinephrine, which is a minor constituent of platelet dense granules (21), also increased acid hydrolase secretion in SPD platelets, this effect was only observed at micromolar levels, far in excess of the nanomolar concentrations present in platelets or in normal plasma. In contrast, the corrective effect of ADP was seen at concentrations similar to, and less than, the concentrations of this nucleotide maximally secreted from platelets.

One conclusion suggested by these findings is that the impairment of acid hydrolase secretion in SPD platelets results from the absence of secretable ADP, and is therefore secondary to the dense-granule deficiency in these platelets. However, since added ADP can potentiate the responses to low concentrations of thrombin in normal platelets also $(28,29)$, we cannot rule out the possibility that the correction of secretion by ADP may be unrelated to the cause of the impairment. Alternatively, the impaired secretion response could be associated with abnormalities in signal transduction processes, or in the lysosomal granule structure itself, which would render the platelets less sensitive to the secretion stimulus.

Several other findings, however, provide further support for a relationship between the impaired acid hydrolase secretion and the absence of secretable ADP: (a) Secretion was not impaired in SPD platelets with lesser deficiencies of densegranule substances, including patients with $\alpha$-granule deficiencies as well, suggesting that the impairment might be specifically associated with the virtual absence of dense-granule constituents in the affected patients. (b) Addition of excess 
ATP, which acts as a specific inhibitor of ADP-mediated responses, to normal platelets produced an impairment of acid hydrolase secretion similar to that in SPD platelets, even in the absence of added ADP. Under these conditions, the most likely target for the inhibitory effects of ATP would be secreted ADP. (c) Both the ATP-induced impairment of secretion in normal platelets and the impairment of secretion in SPD platelets were reversed when a higher concentration of thrombin was used, conditions under which thrombin activates platelets independently of secreted (30) or added (28) ADP.

While ADP was able to correct the impaired acid hydrolase secretion in SPD platelets, the platelet sensitivity to these effects of ADP varied markedly among individual patients (Fig. 6). Correction of this secretion response to normal values required as little as $0.01 \mu \mathrm{M}$ ADP or as much as $10 \mu \mathrm{M}$ ADP in different patients. The reasons for this variation in sensitivity are not clear, but they cannot be related to marked differences in the residual levels of dense-granule ADP present in these patients' platelets (Table I). These variations could, however, reflect differences in ADP interaction with the platelet membrane, or possibly differences in sensitivity to the primary thrombin stimulus, since some relation between the concentration of ADP required for correction and the extent of secretion induced by thrombin alone, in the absence of ADP, seems evident in Fig. 6.

The corrective effect of ADP clearly distinguishes the impairment of acid hydrolase secretion seen in SPD platelets from that which occurs in normal platelets after in vivo, but not in vitro, exposure to ASA. Thus, in platelets exposed to ASA in vivo, ADP $(10 \mu \mathrm{M})$ increased the extent of secretion, but clearly did not normalize this response (Table VI), suggesting that the inhibition by ASA could not be completely ascribed to an inhibition of ADP secretion from dense granules. ASA inhibition of acid hydrolase secretion was associated with, but did not specifically result from, inhibition of cyclooxygenase, since comparable extents of inhibition of $\mathrm{TxB}_{2}$ formation were obtained with both in vivo and in vitro incubation with ASA. Inhibitory effects of ASA, also independent of cyclooxygenase inhibition, have been obtained on rabbit platelet aggregation and dense-granule secretion, but at much higher relative ASA concentrations (31). Neither the inhibition of lysosomal secretion observed here, nor the inhibition of rabbit platelet function (31), appeared to be due to salicylate. Thus, the mechanism of this inhibitory effect of ASA in vivo remains obscure.

The ability of excess ATP, acting as a specific antagonist of ADP (26), to partially inhibit acid hydrolase secretion induced by low concentrations of thrombin in normal platelets (Table IV) suggests that ADP plays a role in the lysosomal secretion response of normal platelets also. Since exogenous ADP did not alter this secretion response in normal platelets, it is reasonable to presume that such effects would most likely occur via ADP secreted from the dense granules. A role for secreted ADP in other platelet responses has been suggested by earlier studies. Thus, e.g., a partial inhibition of dense-granule secretion induced by low concentrations of thrombin, similar to those used in this study, and by A23187 in washed rabbit platelets was observed in the presence of the ADP-removing enzyme system, creatine phosphate-creatine phosphokinase (30). The activation of phospholipase C by A23187 has also been reported to be totally dependent on secreted ADP and cyclooxygenase products (32). In addition, correction by ADP of platelet procoagulant defects was demonstrated in one of the early studies of patients with impaired aggregation responses (33), some of whom were later shown to have SPD (13).

The mechanism by which ADP might mediate acid hydrolase secretion, or whether similar mechanisms govern the effects of ADP on acid hydrolase secretion and on the other platelet responses described above, remains to be defined. The results shown in Table III demonstrate that the effects of ADP on acid hydrolase secretion occur in association with the direct activation of the platelets in suspension, and are not a secondary, "conditioning" effect (23) promoting centrifugation-induced secretion. In addition, the results in Table IV indicate that these effects are dependent on the interaction of ADP with its membrane receptor. Thus, the effects of ADP could reflect simply a synergistic interaction with thrombin or A23187, similar to those described for many other pairs of agonists (29, 34). However, it is important to note a fundamental difference between the effects of ADP on acid hydrolase secretion and the synergistic interactions of pairs of agonists described in the latter two references. As shown in earlier studies (35) as well as in the present studies, ADP alone was incapable of inducing acid hydrolase secretion, and thus cannot be considered an agonist for this response. These effects of ADP are therefore not strictly comparable to synergistic interactions between pairs of agonists but may instead represent potentiation of the thrombin- or ionophore-induced lysosomal secretion response by ADP. This potentiation was most evident as an increased sensitivity of the platelets to lower concentrations of the primary stimulus, and was usually not associated with an increased maximal extent of secretion. A similar pattern of potentiation of thrombin-induced dense granule secretion by epinephrine, under conditions where epinephrine itself does not induce secretion, has been demonstrated by Steen and Holmsen (36). The present studies indicate that epinephrine, which, like ADP, does not induce acid hydrolase secretion itself, also enhances thrombin-induced lysosomal secretion, and preliminary studies (Holmsen, $\mathrm{H}$., unpublished observations) indicate that the dose response curve for this response is also shifted to the left by epinephrine.

These effects of epinephrine, as well as its ability to restore irreversible aggregation in functionally inhibited platelets (22), were observed at micromolar levels of this substance. However, our findings that no potentiation of acid hydrolase secretion occurred at nanomolar levels of epinephrine, comparable to the amounts normally present in plasma or secreted from platelets, indicate that epinephrine potentiation of this response may not be as significant physiologically as potentiation by ADP.

\section{Acknowledgment}

This study was supported in part by grant HL-27346 from the National Institutes of Health.

\section{References}

1. Holmsen, H., and H. J. Weiss. 1979. Secretable storage pools in platelets. Annu. Rev. Med. 30:119-134.

2. Mills, D. C. B., I. A. Robb, and C. C. K. Roberts. 1968. The release of nucleotides, 5-hydroxytryptamine and enzymes from human blood platelets during aggregation. J. Physiol. (Lond.). 195:715-729. 
3. Holmsen, H., C. A. Dangelmaier, and S. Rongved. 1984. Tight coupling of thrombin-induced acid hydrolase secretion and phosphatidate synthesis to receptor occupancy in human platelets. Biochem J. 222:157-167.

4. Holmsen, H., C. A. Setkowsky, B. Lages, H. J. Day, H. J. Weiss, and M. C. Scrutton. 1975. Content and thrombin-induced release of acid hydrolases in gel-filtered platelets from patients with storage pool disease. Blood. 46:131-142.

5. Witte, L. D., K. L. Kaplan, H. L. Nossel, B. A. Lages, H. J. Weiss, and De W. S. Goodman. 1978. Studies of the release from human platelets of the growth factor for cultured human arterial smooth muscle cells. Circ. Res. 42:402-409.

6. Holmsen, H., K. L. Kaplan, and C. A. Dangelmaier. 1982. Differential energy requirements for platelet responses: a simultaneous study of aggregation, three secretory processes, arachidonate liberation, phosphatidylinositol breakdown, and phosphatidate production. Biochem. J. 208:9-18.

7. Verhoeven, A. J. M., M. E. Mommersteeg, and J. W. N. Akkerman. 1984. Quantification of energy consumption in platelets during thrombin-induced aggregation and secretion: Tight coupling between platelet responses and the increment in energy consumption. Biochem. J. 221:777-787.

8. Weiss, H. J., L. D. Witte, K. L. Kaplan, B. A. Lages, A. Chernoff, H. J. Nossel, De W. S. Goodman, and H. R. Baumgartner. 1979. Heterogeneity in storage pool deficiency: studies on granule-bound substances in 18 patients including variants deficient in $\alpha$-granules, platelet factor $4, \beta$-thromboglobulin, and platelet-derived growth factor. Blood. 54:1296-1319.

9. White, J. G. 1986. Platelet granule disorders. CRC Crit. Rev. Oncol./Hematol. 4:337-378.

10. Bell, T. G., K. M. Meyers, D. J. Prieur, A. S. Fauci, S. M. Wolf, and G. A. Padgett. 1976. Decreased nucleotide and serotonin storage associated with defective function in Chediak-Higashi syndrome in cattle and human platelets. Blood. 48:175-184.

11. Boxer, G. J., H. Holmsen, L. Robkin, N. U. Bang, L. A. Boxer, and R. L. Baehner. 1977. Abnormal platelet function in Chediak-Higashi syndrome. Br. J. Haematol. 35:521-533.

12. Rendu, F., J. Breton-Gorius, M. Lebret, C. Klebanoff, D. Buriot, C. Griscelli, S. Levy-Toledano, and J. P. Caen. 1983. Evidence that abnormal platelet functions in human Chediak-Higashi syndrome are the result of a lack of dense bodies. Am. J. Pathol. 111:307-314.

13. Holmsen, H., and H. J. Weiss. 1972. Further evidence for a deficient storage pool of adenine nucleotides in platelets from some patients with thrombocytopathia-"storage pool disease." Blood. 39:197-209.

14. Gerrard, J. M., D. C. Phillips, G. H. R. Rao, E. F. Plow, D. A Walz, R. Ross, L. A. Harker, and J. G. White. 1980. Biochemical studies of two patients with the gray platelet syndrome: selective deficiency of platelet alpha granules. J. Clin. Invest. 66:102-109.

15. Koike, K., A. K. Rao, H. Holmsen, and P. S. Mueller. 1984. Platelet secretion defect in patients with the attention deficit disorder and easy bruising. Blood. 63:427-433.

16. Lages, B., M. C. Scrutton, and H. Holmsen. 1975. Studies on gel-filtered human platelets: isolation and characterization in a medium containing no added $\mathrm{Ca}^{+2}, \mathrm{Mg}^{+2}$, or $\mathrm{K}^{+}$. J. Lab. Clin. Med. 85:811-825.

17. Dangelmaier, C. A., and H. Holmsen. 1980. Determination of acid hydrolases in human platelets. Anal. Biochem. 104:182-191.

18. Weiss, H. J., P. A. Chervenick, R. Zalusky, and A. Factor. 1969. A familial defect in platelet function associated with impaired release of adenosine diphosphate. N. Engl. J. Med. 281:1264-1270.
19. Lages, B., H. Holmsen, H. J. Weiss, and C. Dangelmaier. 1983 Thrombin and ionophore A23187-induced dense granule secretion in storage pool deficient platelets: evidence for impaired nucleotide storage as the primary dense granule defect. Blood. 61:154-162.

20. Blank, L. 1980. Statistical Procedures for Engineering, Management, and Science. McGraw-Hill Book Co., New York.

21. Da Prada, M., and G. B. Picotti. 1979. Content and subcellular localization of catecholamines and 5-hydroxytryptamine in human and animal blood platelets: monoamine distribution between platelets and plasma. Br. J. Pharmacol. 65:653-662.

22. Rao, G. H. R., J. M. Gerrard, C. J. Witkop, and J. G. White. 1981. Platelet aggregation independent of ADP release or prostaglandin synthesis in patients with Hermansky-Pudlak syndrome. Prostaglandins Med. 6:459-472.

23. Massini, P., and E. F. Lüscher. 1971. The induction of the release reaction in human blood platelets by close cell contact. Thromb. Diath. Haemorrh. 25:13-20.

24. Holmsen, H., and C. A. Setkowsky-Dangelmaier. 1977. Adenine nucleotide metabolism of blood platelets. X. Formaldehyde stops centrifugation-induced secretion after A23187 stimulation and causes breakdown of metabolic ATP. Biochim. Biophys. Acta. 497:46-61.

25. Lages, B., and H. J. Weiss. 1980. Biphasic aggregation responses to ADP and epinephrine in some storage pool deficient platelets: relationship to the role of endogenous ADP in platelet aggregation and secretion. Thromb. Haemostasis. 43:147-153.

26. MacFarlane, D. E., and D. C. B. Mills. 1975. The effects of ATP on platelets: evidence against the central role of released ADP in primary aggregation. Blood. 46:309-320.

27. Wiley, J. S., J. Kuchibhotla, C. C. Shaller, and R. W. Colman. 1976. Potassium uptake and release by human blood platelets. Blood. 48:185-197.

28. Packham, M. A., M. A. Guccione, P.-L. Chang, and J. F. Mustard. 1973. Platelet aggregation and release: effects of low concentrations of thrombin or collagen. Am. J. Physiol. 225:38-47.

29. Huang, E. M., and T. C. Detwiler. 1981. Characteristics of the synergistic actions of platelet agonists. Blood. 57:685-691.

30. Kinlough-Rathbone, R. L., M. A. Packham, H. J. Reimers, J. P. Cazenave, and J. F. Mustard. 1977. Mechanisms of platelet shape change, aggregation, and release induced by collagen, thrombin, or A23187. J. Lab. Clin. Med. 90:707-719.

31. Buchanan, M. R., J. A. Rischke, and J. Hirsh. 1982. Aspirin inhibits platelet function independent of the acetylation of cyclo-oxygenase. Thromb. Res. 25:363-373.

32. Rittenhouse, S. E. 1984. Activation of phospholipase C by ionophore A23187 is totally dependent upon cyclo-oxygenase products and ADP. Biochem. J. 222:103-110.

33. Weiss, H. J. 1967. Platelet aggregation, adhesion and ADP release in thrombopathia (platelet factor 3 deficiency)-a comparison with Glanzmann's thrombasthenia and von Willebrand's disease. Am. J. Med. 43:570-578.

34. Grant, J. A., and M. C. Scrutton. 1980. Positive interaction between agonists in the aggregation response of human blood platelets: interaction between ADP, adrenaline and vasopressin. Br. J. Haematol. 44:109-125.

35. Day, H. J., and H. Holmsen. 1971. Concepts of the blood platelet release reaction. Ser. Haematol. IV:3-27.

36. Steen, V. M., and H. Holmsen. 1985. Synergism between thrombin and epinephrine in human platelets: different dose-response relationships for aggregation and dense granule secretion. Thromb. Haemostasis. 54:680-683. 